\title{
A Provenance-based Approach to Support Cognitive Training of the Elderly Using Serious Games
}

\author{
Leonardo Nardi ${ }^{1}$, Daniela Trevisan ${ }^{1}$, Daniel de Oliveira ${ }^{1}$ \\ ${ }^{1}$ Instituto de Computação - Universidade Federal Fluminense (UFF) \\ Av. Gal. Milton Tavares de Souza, s/nº - São Domingos - Niterói - RJ - CEP 24210-346 \\ Inardi@id.uff.br, \{daniela, danielcmo\}@ic.uff.br
}

\begin{abstract}
It is a well-known fact that cognitive skills tend to decline in elderly people. There are several approaches that try to reduce the cognitive losses. One of the most prominent is cognitive training through serious games in several game sessions. This paper introduces a data model and a database for storing computer-based cognitive training data. Using this database, researchers have an integrated database that allows for extracting useful information that can be input for statistical analysis and further data mining tasks. In order to evaluate the feasibility of the proposed approach we have performed a statistical analysis on the evaluations of the "Eye for Detail" game stored in the proposed database. Results reinforced the potential of the database as a rich source of information.
\end{abstract}

\section{Introduction}

Cognitive skills tend to decline as the age of the elderly people progress [Williams and Kemper 2010]. Memory and speed of reaction on events are the most impacted skills. Elderly people who have severely affected cognitive skills tend to depend more on third parties for their daily activities and duties as locomotion, basic needs, etc. Based on this, there are several ways to improve the cognitive skills of the elderly or, at least, to delay the loss of cognition.

Some of these improvements are pharmacological [Maier et al. 2015] and others based on stimuli [Klingberg 2010]. Although both ways present positive results, in this paper, we are especially interested in approaches based on response to stimuli, and more specifically, in cognitive training. Cognitive training involves the design and execution of a set of tasks, where each task is associated with a specific cognitive skill (e.g. attention). Cognitive training tasks can be implemented in several ways, but one of the most prominent forms has been the use of computer games [Basak et al. 2008]. In this type of training, elderly people are presented to a series of games and should play a sequence of game sessions, where each session aims at stimulating a different cognitive skill [Kelly et al. 2014].

Despite the proven efficacy in improving cognitive abilities in the elderly [Netto 2010], most of the cognitive training still present room for optimization of cognitive training process. For example, in many trainings there is no structured way to store and query the data collected by the studies, from the elderly people's personal information and history, anamnesis, game results, etc. If researchers were able to query such data, they could identify problems or patterns and use such knowledge to improve the entire process.

However, querying the data is far from trivial in the current scenario. Since researchers are not computer science experts, they use multiple spreadsheets to represent 
such data. The use of spreadsheets, although functional, presents a number of problems when compared to the use of more robust approaches such as Database Management Systems (DBMS), such as data integrity, data redundancy control, scalability, query performance and security.

In addition, since all data is historical and associated to elderly people and a series of events (game sessions), it can be characterized as Provenance data [Missier et al. 2013]. Provenance can be defined all information about entities (e.g. games), activities (e.g. game sessions, evaluations), and people (e.g. elderly people) involved in producing a piece of data, which can be used to form assessments about its quality, reliability or trustworthiness. Since supporting evaluating the quality, reliability and trustworthiness of the cognitive training is our goal, all data should be represented according to Provenance data standards defined by W3C (https://www.w3.org/TR/provoverview/).

This way, it is fundamental to develop a conceptual model that can be used to create a database that contains information related to tests performed with elderly before and after the accomplishment of game-based cognitive trainings. This conceptual model enables querying and extracting data for analytics and application of statistical tests to search for patterns that show the effectiveness of computer games in the cognitive training of the elderly.

This paper focuses on the representation (in a provenance database) and the statistical analysis of data from sessions of computer cognitive training. In our case study, we have chosen the game "Eye for Detail" of the "Brain Speed" game group and the RAVLT exam (that evaluates the results of "Brain Speed" games), which informs the CR (Correctly Rejected) and Hits (Recognition) scores as performance measures. We observed these scores along 40 hours of cognitive training and compare the results with a control group.

This paper is organized in 6 sections besides this Introduction. Section 2 introduces important concepts associated with Cognitive Training, the "Eye for Detail" game (case study in this paper) and Provenance. Section 3 presents the provenance database that was modelled. Section 4 brings the experimental results. Section 5 discusses related work. Section 6 concludes the paper and points out future work, and, finally, Section 7 brings the acknowledgment.

\section{Background Knowledge}

This section presents important concepts that we have applied in our research methodology for investigate cognitive training with games.

This section starts defining cognitive training and then presents the "Eye for Detail" game, which is used as case study in this paper. In addition, it defines provenance and the PROV standard [Missier et al. 2013] used in the conceptual model.

There are many others approaches involving cognitive training with games and these approaches are presented later in the section of Related Works. 


\subsection{Computer-based Cognitive Training}

All cognitive training procedures presented in this section follow the procedures defined by the research group coordinated by the physician and neuroscientist Prof. PhD Rogério Panizzutti, from the Institute of Biomedical Sciences (IBS) and the Institute of Psychiatry (IPUB) of the Federal University of Rio de Janeiro. IPUB is composed of a multidisciplinary team of psychologists, in physical education and students in related fields. This team performs computer-based cognitive training in Applied Psychology Service (SPA) of the Department of Psychology at the Pontifical Catholic University of the State of Rio de Janeiro.

The cognitive training consists of weekly sessions of games (usually played 3 times a week), totaling 40 hours over 3 to 6 months depending on the subject's frequency. A total of 21 subjects are currently participating in training with a minimum age of 60 years and a maximum of 86 years ( 73 years average). All games that each subject interacts are part of BrainHQ platform [PositScience 2018] and are organized in several groups named "Attention games", "Brain Speed", "Memory", "People Skills", "Intelligence" and "Navigation". The group of games as well as the order of execution is defined by the team of researchers.

The evaluation model of the elderly is composed of 4 phases named screening, pre-intervention (AV1), intervention (AV2) and post-intervention (AV3). In screening, the subject is evaluated for vision, hearing, cognition (Mini-Mental Exam), intelligence quotient (IQ), depression, and functionality. Subjects that considered healthy, after screening, are subject to the AV1, AV2 and AV3 evaluations in which factors of quality of life, memory, processing speed, attention/concentration, executive function, social cognition, praxis and physical activity are analyzed. Following we provide more details of each assessment (AV1, AV2 and AV3).

\subsubsection{Assessments}

The assessments evaluate the participants before, during and at the end of computer-based cognitive training. They are divided into: pre-intervention (AV1), intervention (AV2) and post-intervention (AV3).

After the screening participants are submitted to a first evaluation (AV1) or preintervention. In it, the participants obtain, for each associated exam, a certain score. Subsequently, participants are divided into two categories: computer-based brain training and commercial computer games or control (placebo). After 20 hours of computer-based cognitive training, participants are re-evaluated through a second assessment (AV2) or intervention. In it, participants obtain for each associated exam, a new score. At the end of the remaining 20 hours of training, totaling 40 hours, since the last evaluation (AV2), participants are submitted to interventional intervention (AV3). In it, participants obtain for each associated exam, a new score.

In this study we are focusing on the "Eye for Detail" that is evaluated by applying the RAVLT exam for each evaluation. We detail them following in the next sections. 


\subsection{Eye for Detail Computerized Cognitive Game}

(Figure 1) belongs to the "Brain Speed" group and aims at stimulating visual processing speed and visual working memory by focusing 3 images displayed at the beginning and then in advance displays 5 images to be focused by the elderly participant. Both images will appear in different positions on the screen, briefly flashed. As the game progresses, the images become similar, requiring the older participant a greater focus. Moreover, as the participant progresses, during the game, getting a score in milliseconds, images flash faster. Table 1 presents the main characteristics of the "Eye for Detail" game, including the "Action" performed by the subject, "Targeted Skills", "How the exercise changes" and "How you are scored".

Table 1. Eye for Detail Game Descriptions [PositScience 2018]

\begin{tabular}{|l|l|l|l|}
\hline What you do & Targeted skills & $\begin{array}{l}\text { How the exercise } \\
\text { changes }\end{array}$ & $\begin{array}{l}\text { How you are } \\
\text { scored }\end{array}$ \\
\hline $\begin{array}{l}\text { Watch as three or } \\
\text { five images briefly } \\
\text { flash in different } \\
\text { positions on screen }\end{array}$ & $\begin{array}{l}\text { Visual processing } \\
\text { speed }\end{array}$ & $\begin{array}{l}\text { The images } \\
\text { change, becoming } \\
\text { more similar and } \\
\text { they are shown } \\
\text { over a larger area } \\
\text { on screen }\end{array}$ & $\begin{array}{l}\text { Your score is in } \\
\text { milliseconds }\end{array}$ \\
\cline { 2 - 4 } & $\begin{array}{l}\text { Vou go from } \\
\text { viewing 3 images } \\
\text { to 5 images }\end{array}$ & $\begin{array}{l}\text { As you improve, } \\
\text { the images flash on } \\
\text { screen for fewer } \\
\text { milliseconds. }\end{array}$ \\
\hline
\end{tabular}

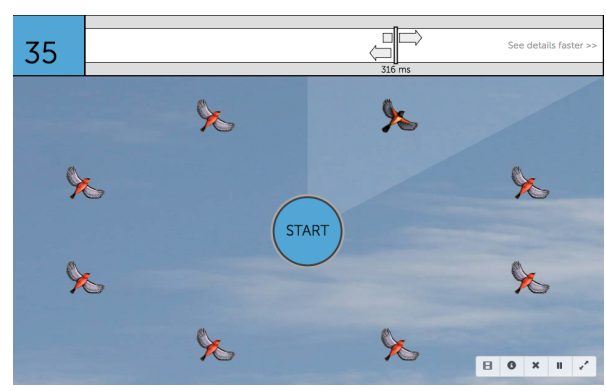

Figure 1. The "Eye for Detail" Game Screen.

\subsection{RAVLT exam (CR and Hits categories)}

The RAVLT is the exam used to evaluate the results of "Eye for Detail" game. RAVLT (Rey Auditory Verbal Learning Test) [BOONE et al. 2005] to be an instrument that belongs to memory category and be applied in all evaluations (AV1, AV2 and AV3), is an effective test that measures how an elderly participant improved (or not) his/her performance and therefore his/her memory, based on computer-based cognitive training. For this reason, we have chosen this test as case study for this paper since we can apply the statistical analyzes in the groups of elderly participants who interacted with the computerbased cognitive games. 
As noted previously the RAVLT [BOONE et al. 2005] exam is performed by an appraiser who read 15 words in a paused way and at the end of the reading, ask the subject to repeat them in the same order or not. The purpose of this test is for the subject to be able to memorize as many words as possible.

From this exam, the categories CR and Hits are considered that are the most relevant to measure the performance and, thus, the improvement in the subject's cognition. In the Hits category, the evaluator reads to the participant 50 words and, for each word, ask him/her if the word is on the list or not. As the participant is hitting the list of items that he/she memorized earlier, the participant earns one point. The maximum score is 15 , since there are 15 items in the list.

In the CR category, the evaluator subtracts the 50 words by the total number of correct words that the participant previously memorized. This results in $35(50-15)$ words that do not belong the list of 15 words. Thus, the CR is equal to 35 subtracted by false positives, i.e., words that the participant classified as correct. For example, of the 35 words that do not belong, the participant said that 2 words were correct (they belonged to the 15 words previously memorized), generating in a false positive that resulted in 35 words minus 2 false positives equal to 33 words correctly rejected by the participant. If the participant answers correctly based on the 15 memorized words, it does not produce false positives and therefore all 35 words are Correctly Rejected (CR).

\subsection{Provenance and PROV Standard}

The term "Provenance" can be defined in several ways. But amongst all existing definitions, the one that interests us in the context of this paper is "origin". Provenance aims at registering the origin and history of an information. It allows for analyzing the procedures performed and the data consumed and produced are validated and reproducible by third parties. Thus, Buneman et al. [Buneman et al. 2001] were the first authors to define the question of the origin of data in scientific procedures, defining the term as the description of the origin of a data and the process by which it stored in a database.

In general, the provenance can be characterized in two ways: prospective and retrospective [Lim et al. 2010]. The prospective provenance is interested in capturing and storing data on the process structure that led to the generation of a particular product (the scientific procedure). That is, in the context of this paper, the prospective provenance is concerned with the games, the subjects, etc. The retrospective provenance focuses on capturing the data and its descriptors produced from the execution of a certain activities. Retrospective provenance data includes start and end times, scores in a game session, errors that occurred, etc.

In order to store the retrospective and prospective provenance data, data models should follow the W3C PROV standard [W3C 2018]. PROV proposes a generic and abstract representation of provenance. The main idea behind PROV is to represent the most important concepts of provenance that can be specialized for different domains. Provenance is information about entities, activities, and people involved in producing a piece of data, which can be used to form assessments about its quality, reliability or trustworthiness as discussed in the Introduction. PROV is a conceptual data model that forms a basis for the W3C provenance (PROV) family of specifications (that includes data models, ontologies, scripts, etc.). The PROV data model is composed by 3 classes: agent, entity and 
activity as presented in Figure 2.

According to PROV standard an entity is "a physical, digital, conceptual, or other kind of thing with some fixed aspects; entities may be real or imaginary". In the context of this paper, a game can be considered as an entity. An activity can be also defined as "something that occurs over a period of time and acts upon or with entities; it may include consuming, processing, transforming, modifying, relocating, using, or generating entities". A game session can be considered an activity in the context of this paper. And, finally, an agent is "something that bears some form of responsibility for an activity taking place, for the existence of an entity, or for another agent's activity". In the context of this paper, an agent can be a specific subject.

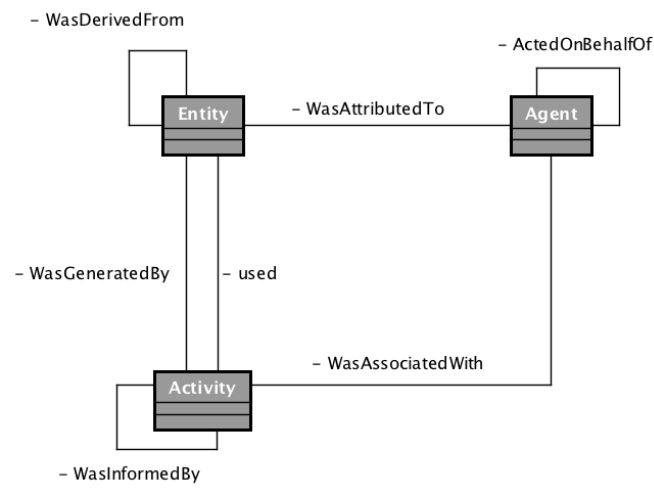

Figure 2. The PROV-DM Class Diagram [W3C 2018]

One of the main advantages of using standards and recommendations such as PROV is to ensure the interoperability of provenance descriptors from heterogeneous environments, regardless of the technology and approach used.

\section{Proposed approach: a provenance model and database for suppporting cognitive training}

As aforementioned, this paper presents a research that involves 2 main areas of computer science: modeling and data extraction of data from a database containing evaluations of computer-based cognitive training using serious games. Our focus in this paper is to present the developed model and the extraction of relevant information, from the modeled database, pre- and post-interaction of the elderly subjects in the computer-based cognitive games associated to the problem. After that, we apply a statistical test that demonstrates that the database is queryable and useful for checking if subjects did or did not improve cognitive performance.

This section presents CogTraining-DM (Cognitive Training Data Model), a conceptual model for representing cognitive training data for the elderly and its instantiation in a Relational Database. CogTraining-DM specializes the PROV-DM, thus it can be considered PROV compliant, i.e. provenance can be easily extracted from this model for further analysis. The development process of CogTraining-DM has four stages: (i) The conceptual modeling, at a high abstraction level, of the stages of the evaluations (screening, AV1, AV2 and AV3) and the data of their respective tests previously discussed in the Section II; (ii) Instantiation of the database following the proposed conceptual model; 
(iii) Development of an algorithm to ease the ETL (extract, transform and load) process from several spreadsheets and thus to store this information in tables of the provenance database and (iv) Development and generation of graphics as a result the extraction of relevant information from the interaction of the elderly participants in the computer-based cognitive training for statistical tests.

For the design of CogTraining-DM, the Unified Modeling Language (UML) and the Astah software were used. The UML was chosen for conceptual modeling as it aims to specify, visualize, design and document the software artifacts as described by their creators. [Booch et al. 2005]. An excerpt of the CogTraining-DM is presented in Figure 3.

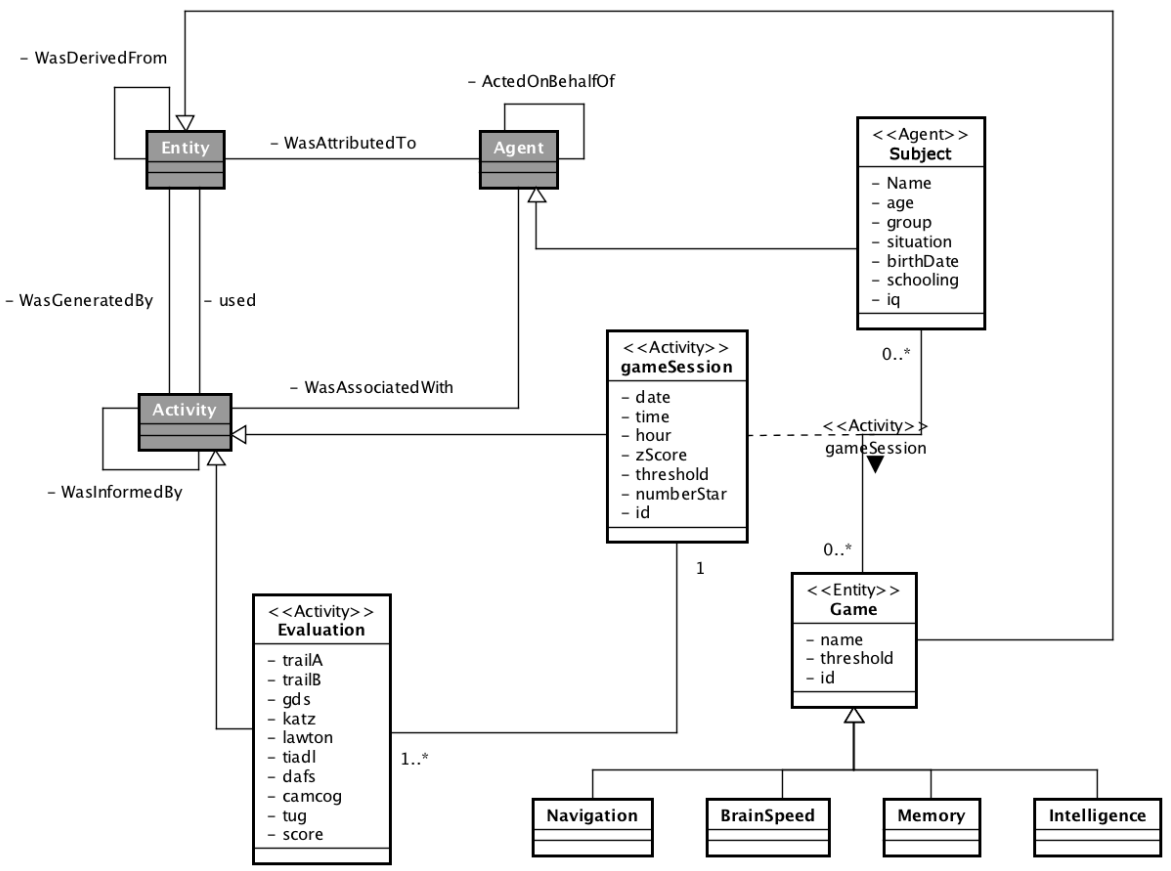

Figure 3. An Excerpt of the Proposed CogTraining-DM.

Analyzing Figure 3 we can see the main classes of the proposed model (in white): Subject, Game, gameSession and Evaluation. Subject stores information related to each subject such as IQ level, name, age, etc. Game stores information about the serious game involved in the cognitive training. The associative class gameSession stores information about the game sessions played by subjects. Each game session is associated to a subject and a game. Each subject can play many game sessions. Evaluation stores information about the evaluations performed (AV1, AV2 and AV3) for each game. Based on the results of the evaluations we can extract useful information for further data analytics.

It is worth noticing that the main classes of CogTraining-DM (in white) extend one class of the PROV-DM (in dark gray). It means that each class associated to cognitive training specializes one of the PROV concepts. Thus, it is intuitive and simple to extract Agents, Entities and Activities from CogTraining-DM. In addition, we can benefit from the several existing tools for managing and sharing provenance data that are based on the PROV model.

After defining a high level conceptual model (CogTraining-DM) that encompasses 
the main concepts of the domain, a physical database project was then elaborated, based on this conceptual model, and thus created a database with the name "Brain Academy". The design of this database was performed by means of the high-level mapping of each entity, agent and activity of the conceptual model for a representation in tables in the physical database and, therefore, managed by a Database Management System (DBMS). We used PostgreSQL database version 9.6 as DBMS. This Software, besides being free, is one of the DBMSs widely used in academic research.

The development of an algorithm was necessary to ease the capture of the data that was dispersed in several spreadsheets and thus to extract, transform, and load data to the database tables. The design of the algorithms followed the principles and techniques Object Orientation to ease loading task, since it is a tedious and error-prone task if manually performed. Furthermore, due to space restrictions, the loading scripts cannot be placed in the paper, but they can be downloaded at our group GitHub repository (github.com/UFFeScience/Cognitive-Game-Analytics).

After all data is loaded in the database, the researcher is able to perform SQL queries to extract desired data. Following, we present a query that aims at extracting all information from evaluations performed over game sessions of a fictional subject "Amália Rodrigues" when playing the game "Eye for Detail". As shown in the Figure 4.

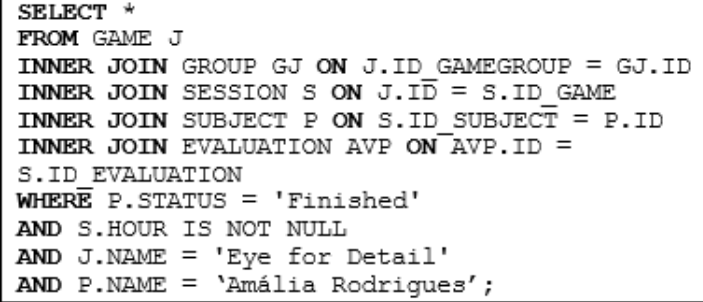

Figure 4. Query Example for an Elderly Participant.

\section{Experimental Results}

In order to evaluate the feasibility of the CogTraining-DM and the database to store data and to allow for extraction we performed an analytics experiment. The results presented following in this section consider the interaction of elderly participants with the "Eye for Detail" game (extracted using the SQL query presented in the previous section), from the "Brain Speed" game group. These results were divided into three moments of evaluations AV1-AV2, AV2-AV3 and AV1-AV3. Each evaluation compares the evolution of the subject from one RAVLT exam to another. For example, group AV1-AV2 analyzes the evolution of the subject from AV1 to AV2. The subjects were divided into three training groups named $\mathrm{A}, \mathrm{B}$ and $\mathrm{C}$.

The "A" group consisted of 3 elderly participants with average IQ $=115$ and the average age $=72$, with a 20-hour computer-based cognitive training. During this 20hour period, the subjects played several games, not only "Eye for Detail". In the AV1AV2 evaluation the participants had no previous contact with any other computer-based cognitive game.

The "B" group included the participation of 8 elderly individuals with an average IQ $=111.75$ and average age $=74.62$, with an additional 20-hour computer-based cog- 
nitive training. During this 20-hour period, the subjects played several games, not only "Eye for Detail". The difference between the AV1-AV2 and AV2-AV3 evaluations is that in the AV2-AV3 evaluation the participants had already performed an AV1-AV2 game session with "Eye for Detail".

For the "C" group, the participant performed AV1-AV2 and AV2-AV3 evaluations, totalizing 40-hour computer-based cognitive training with greater contact with the "Eye for Detail" game during 40 hours of training. Six elderly people participated in this training, with average IQ $=110.33$ and average age $=75$.

Elderly participants in the control group (D group), placebo or commercial computer games, due to their nature, also performed all evaluations, AV1-AV2 (20 hours of training), AV2-AV3 (20 hours of training) and AV1-AV3 (40 hours of training). These participants presented average IQ $=110.57$ and the average age $=74.14$.

Results of CR and Hits mean scores for each group are shown in Figure 5 and Figure 6.

Looking the CR scores in Figure 5 we can observe that playing only 20 hours of Eye for Detail (A and B groups) cannot be enough to increase the CR scores. "C" group which played the game along 40 hours got better cognitive answer.

Looking the Hits scores in Figure 6 we can observe that only 20 hours of Eye for Detail can be enough to reach good Hits scores. "A" group which played the game in the first 20 hours of training increased its Hits score from AV1-AV2 to AV2-AV3. "B" group which played the game in the last 20 hours of training increased its Hits score from AV2AV3 to AV1-AV3. "C" group which played the game along 40 hours of training reached better Hits score in the first 20 hours.

Since the number of samples in each group is small and the group are independents we applied the non-parametric Mann Whitney U Test in order to investigate the statically significance difference in our results. We found difference only for CR scores between C group with rank mean=14,83 and D group (control group) with rank mean $=8,64$ and $\mathrm{U}=68$ and $\mathrm{p}=0.033$. With that we can say participants who played more time the game Eye for Detail during the cognitive training reached better CR scores than the control group.

\section{Related Work}

Analysis and understanding of events, errors, and flows of a particular game session can be useful for a variety of reasons: to understand problems related to gameplay, data mining of specific situations, and even understanding the educational, cognitive and learning aspects of serious games. In this context Consalvo et al. [Consalvo and Dutton 2006] presents a formal approach based on metrics collected during the user game session and proposes a template for data analysis. Playtracer [Andersen et al. 2010] allows for a more detailed visual analysis to identify the decisions made by the players during the game session.

Cognitive control is defined by a set of neural processes that allow us to interact in our complex environment in a direct way. Humans beings regularly challenge these control processes while attempting to simultaneously accomplish multiple (multitasking) goals, generating interference as a result of limitations in processing key information. In this context, Anguera et al. [Anguera et al. 2013] presents an approach in which it 


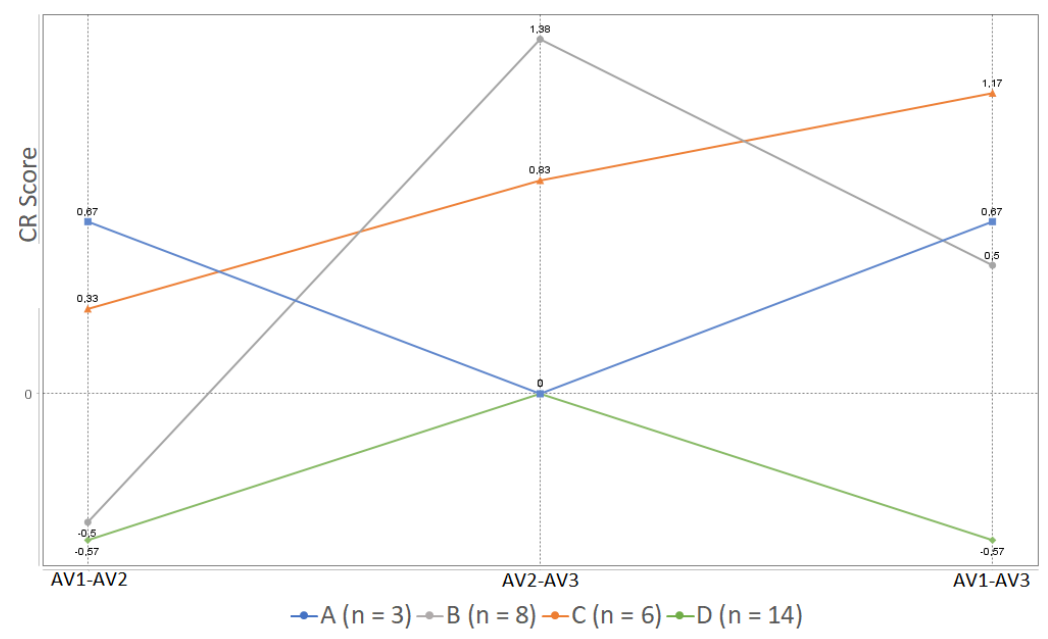

Figure 5. CR Scores of Elderly Participants for "Eye for Detail" Game

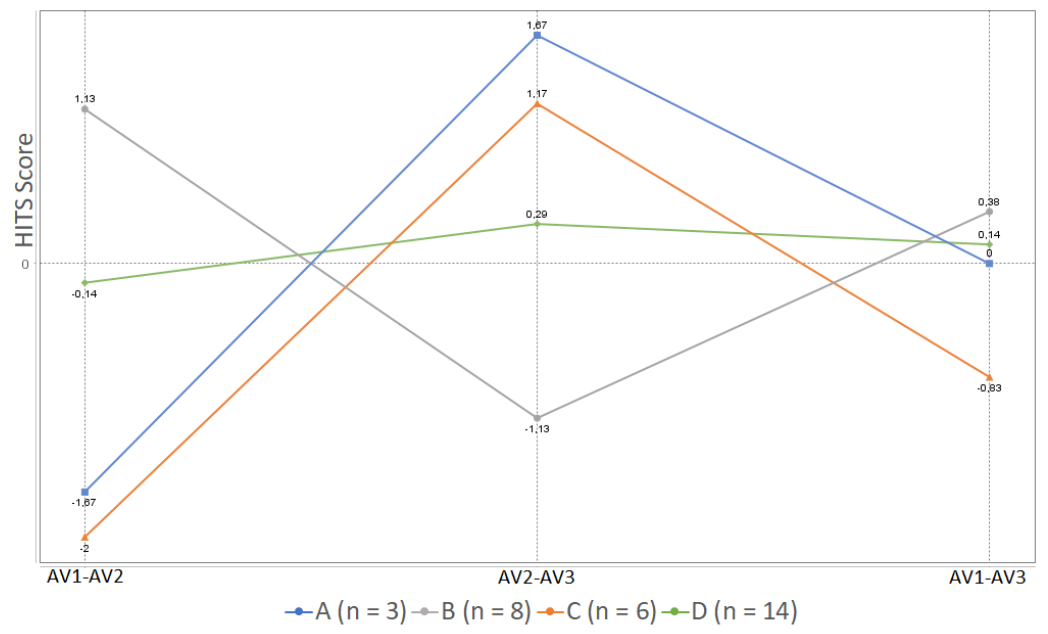

Figure 6. HITS Scores of Elderly Participants for "Eye for Detail” Game

makes use of a customized three-dimensional video game (NeuroRacer) and an adaptive version of it in the multitasking training mode applied to groups aged 20 to 79 years and groups aged 60 to 89 years. In this approach Anguera et al. [Anguera et al. 2013] pointed out that the plasticity of the prefrontal cognitive control system in brain aging provides the first evidence, to our knowledge, of how a personalized video game can be used to evaluate cognitive abilities throughout life, to evaluate the underlying neural mechanisms and serve as a powerful tool for cognitive enhancement.

The interaction of the elderly participant in a computer-based cognitive training through serious games that stimulates their memory, attention, cognition among others is of paramount importance to prevent the participant from possible degenerative diseases, such as Alzheimer's. In this context, Chi, Agama, and Prodanoff [Anguera et al. 2013] propose the design of a computer-based serious game suite called the "Smart Thinker", which is used to enhance core cognitive skills focusing specifically on attention and memory skills. Thus, the authors selected 59 elderly who were separated randomly into control 
group and intervention group. Finally, they applied the Mini Mental State Examination (MMSE) in both groups at the beginning and end of the 6-week period and then applied a statistical t-test with Levene's test to see which of the groups performed best. It is worth mentioning that our approach can be used with different games, including the ones proposed by Chi, Agama, and Prodanoff [Chi et al. 2017].

\section{Conclusions and Future Work}

This paper focused on proposing a data model and a database that stores information regarding computer-based cognitive training using serious games. Based on the stored data, researchers can extract useful information and then perform some statistical analysis. In this paper, we used as case study the "Eye for Detail" game and the RAVLT exam (CR and Hits scores), applying the statistical measures for performance analysis, in two distinct assessment groups and based on the scores extracted for that set of samples. With that we can say participants who had more contact with the game got better results for CR scores indicating the efficacy of the cognitive training. However more tests with others games from the Brain Speed category are needed in order to verify this assumption.

Although this paper has restricted the analysis of the cognitive serious game "Eye for Detail" for the Hits (recognition) and CR (Correctly Rejected), the results obtained demonstrated the effectiveness of computer-based cognitive training as a preventive measure against degenerative diseases of the brain, such as Alzheimer, which reaches about 44 million people worldwide [Alzheimers.net 2018]. In addition, the experiments reinforced the suitability of the proposed model and shows that it open room for computer-based cognitive training analytics.

As future work, we plan to investigate the interaction of the elderly participant with other computer-based cognitive serious games, not addressed in this paper. Furthermore, we plan to conduct the performance analysis for each survey mapped to these games. In this way, it will be possible to identify which game presents better performance in relation to the stimulation of brain neuroplasticity.

\section{Acknowledgment}

Authors would like to thank CNPq, CAPES and FAPERJ for partially sponsoring this research. Authors would like to thank Yasmin Guedes de Oliveira from PUC-Rio for providing the subjects data for our modeling analysis.

\section{References}

Alzheimers.net (2018). 2017 alzheimer's statistics. http://www.alzheimers.net/resources/alzheimers-statistics/. March 26, 2018.

Andersen, E., Liu, Y.-E., Apter, E., Boucher-Genesse, F., and Popović, Z. (2010). Gameplay analysis through state projection. In Proceedings of the Fifth International Conference on the Foundations of Digital Games - FDG '10, pages 1-8, New York, New York, USA. ACM Press.

Anguera, J. A., Boccanfuso, J., Rintoul, J. L., Al-Hashimi, O., Faraji, F., Janowich, J., Kong, E., Larraburo, Y., Rolle, C., Johnston, E., and Gazzaley, A. (2013). Video game training enhances cognitive control in older adults. Nature, 501(7465):97-101. 
Basak, C., Boot, W. R., Voss, M. W., and Kramer, A. F. (2008). Can training in a realtime strategy video game attenuate cognitive decline in older adults? Psychology and aging, 23(4):765-77.

Booch, G., Rumbaugh, J., and Jacobson, I. (2005). UML Guia do Usuário. Elsevier, 2 ed. edition.

BOONE, K., LU, P., and WEN, J. (2005). Comparison of various RAVLT scores in the detection of noncredible memory performance. Archives of Clinical Neuropsychology, 20(3):301-319.

Buneman, P., Khanna, S., and Tan, W.-C. (2001). Why and Where: A Characterization of Data Provenance. In ICDT 2001: Database Theory, pages 316-330, London, UK.

Chi, H., Agama, E., and Prodanoff, Z. G. (2017). Developing serious games to promote cognitive abilities for the elderly. In 2017 IEEE 5th International Conference on Serious Games and Applications for Health (SeGAH), pages 1-8. IEEE.

Consalvo, M. and Dutton, N. (2006). Game analysis: Developing a methodological toolkit for the qualitative study of games. Game Studies: the international journal of computer game research, 6(1).

Kelly, M. E., Loughrey, D., Lawlor, B. A., Robertson, I. H., Walsh, C., and Brennan, S. (2014). The impact of cognitive training and mental stimulation on cognitive and everyday functioning of healthy older adults: A systematic review and meta-analysis. Ageing Research Reviews, 15:28-43.

Klingberg, T. (2010). Training and plasticity of working memory. Trends in Cognitive Sciences, 14(7):317-324.

Lim, C., Lu, S., Chebotko, A., and Fotouhi, F. (2010). Prospective and Retrospective Provenance Collection in Scientific Workflow Environments. In 2010 IEEE International Conference on Services Computing, pages 449-456. IEEE.

Maier, L. J., Liakoni, E., Schildmann, J., Schaub, M. P., and Liechti, M. E. (2015). Swiss University Students' Attitudes toward Pharmacological Cognitive Enhancement. PLOS ONE, 10(12).

Missier, P., Belhajjame, K., and Cheney, J. (2013). The W3C PROV family of specifications for modelling provenance metadata. In Proceedings of the 16th International Conference on Extending Database Technology - EDBT'13, page 773, New York, New York, USA. ACM Press.

Netto, T. M. (2010). Treinamento cognitivo da memória de trabalho em adultos idosos. Psicologia Clínica, 22(2):218-218.

PositScience (2018). Brain training that works. https://www.brainhq.com/welcome. March 26, 2018.

W3C (2018). Prov-dm: The prov data model. https://www.w3.org/TR/prov-dm/. March $26,2018$.

Williams, K. N. and Kemper, S. (2010). Interventions to reduce cognitive decline in aging. Journal of psychosocial nursing and mental health services, 48(5):42-51. 